\title{
ESTEROIS COMO INDICADORES DO ACÚMULO DE ESGOTOS DOMÉSTICOS EM SEDIMENTOS DE UM SISTEMA ESTUARINO-LAGUNAR TROPICAL (MUNDAÚ-MANGUABA, AL)
}

\author{
Michelle Passos Araujo, Talitha Lopes Ferreira da Costa e Renato da Silva Carreira* \\ Faculdade de Oceanografia, Universidade do Estado do Rio de Janeiro, R. São Francisco Xavier, 524, 20550-013 Rio de Janeiro \\ - RJ, Brasil
}

Recebido em 05/01/10; aceito em 19/7/10; publicado na web em 16/11/10

\begin{abstract}
STEROLS AS INDICATORS OF SEWAGE ACCUMULATION IN SEDIMENTS OF A TROPICAL ESTUARINE-LAGOON SYSTEM (MUNDAÚ - MANGUABA, AL). The history of sewage contamination in the Mundaú-Manguaba estuarine lagoon system (NE Brazilian coastal zone) was evaluated through the concentration of sterols in sediment cores. The concentration of coprostanol increased towards the surface sediments, with the maximum of $5.65 \mu \mathrm{g} \mathrm{g}^{-1}$ at $0-2 \mathrm{~cm}$ sediment layer in Mundaú. Manguaba exhibited a lower level of contamination. The ratio cholestanol/cholesterol suggested degradation of coprostanol only before the burial of organic matter in the sediment. This feature, together with information of population growth in the watershed, allowed the estimation of a sedimentation rate of $0.90 \mathrm{~cm}_{\text {year }}{ }^{-1}$ to the Mundaú lagoon.
\end{abstract}

Keywords: fecal contamination; sediment core; coprostanol.

\section{INTRODUÇÃO}

Os esgotos domésticos são um dos principais vetores de contaminação para ambientes aquáticos, devido ao grande volume produzido diariamente e por conter, além de matéria orgânica, diversas outras classes de contaminantes presentes em produtos de uso residencial, tais como metais, hidrocarbonetos e compostos orgânicos persistentes. ${ }^{1}$ A grande concentração populacional na zona costeira, aliada à falta de infraestrutura para coleta, tratamento e disposição final de esgotos domésticos resulta no lançamento desses efluentes diretamente nos corpos d'água localizados na zona de transição continente-oceano, trazendo prejuízos para a qualidade da água e dos sedimentos, com reflexos sobre a saúde humana. ${ }^{2}$

As bactérias do grupo dos coliformes são indicadores tradicionais da contaminação fecal, sendo largamente utilizadas para atestar condições de balneabilidade dos corpos d'água. ${ }^{3,4}$ No entanto, para acompanhar a dispersão espacial e o acúmulo de esgotos domésticos na zona costeira, é necessário considerar um indicador que tenha maior persistência no meio do que a apresentada pelos indicadores biológicos. Uma das melhores alternativas para contornar esse problema é o uso de moléculas específicas, conhecidas genericamente como marcadores moleculares antropogênicos. ${ }^{5,6}$ Neste sentido, o coprostanol ( $5 \beta$-colestan- $3 \beta$-ol), um esterol de origem fecal produzido no intestino de animais de sangue quente e que representa até $60 \%$ dos esterois fecais em humanos, ${ }^{7}$ tem sido largamente utilizado como indicador da contaminação fecal já desde a década de $1960 .^{6}$

No Brasil, os estudos que usam coprostanol e outros esterois fecais na avaliação da contaminação fecal em áreas costeiras são ainda relativamente recentes, sendo a grande maioria deles relativos às regiões sul-sudeste do país. ${ }^{8-15}$ No geral, esses trabalhos confirmam a aplicabilidade do coprostanol como indicador fecal em regiões tropicais. Por outro lado, sugerem que a geoquímica dos esterois fecais pode ser afetada pela elevada atividade microbiana em altas temperaturas e, desta forma, os índices de contaminação fecal definidos para regiões temperadas ${ }^{6,16}$ devem ser aplicados com cautela em regiões tropicais.

\footnotetext{
*e-mail: carreira@uerj.br
}

No presente trabalho, os esterois coprostanol, colesterol, colestanol e dinosterol foram considerados em testemunhos de sedimento coletados no complexo estuarino-lagunar de Mundaú-Manguaba, AL, com dois objetivos fundamentais: identificar o acúmulo de esgotos domésticos em sedimentos recentes de uma região costeira relativamente pouco estudada, a despeito dos sinais de alterações ambientais associadas ao crescimento urbano desordenado e às mudanças no uso do solo na bacia de drenagem nas últimas décadas; avaliar a eficácia do coprostanol como indicador da contaminação fecal em um sistema retentor de materiais e que, ao mesmo tempo, apresenta um alto potencial de degradação da matéria orgânica devido ao elevado metabolismo microbiano. ${ }^{17-20}$

\section{PARTE EXPERIMENTAL}

\section{Área de estudo}

O complexo estuarino-lagunar de Mundaú-Manguaba (CELMM) está localizado no estado de Alagoas entre as latitudes 9³5' - 946' $\mathrm{S}$ e $35^{\circ} 44^{\prime}-35^{\circ} 58^{\prime} \mathrm{W}$ (Figura $1 \mathrm{~S}$, material suplementar). A lagoa Mundaú (24 km² de área total; $1,5 \mathrm{~m}$ de profundidade) e a lagoa Manguaba (43 km² de área total; $2 \mathrm{~m}$ de profundidade média) são conectadas com o mar por um sistema de canais que engloba uma área total de $12 \mathrm{~km}^{2}$ e termina dentro de um único canal de entrada com o oceano. ${ }^{17,20}$

O clima é tropical e semiúmido, com as estações seca (setembro a março) e úmida (abril a agosto) bem definidas. As marés são semidiurnas com amplitude de aproximadamente $1,44 \mathrm{~m}$, sendo que o efeito dessa variação é bastante reduzido no interior das lagoas, particularmente em Manguaba. Desta forma, a lagoa Mundaú é mais influenciada pelas correntes de maré e, por isto, o tempo de renovação das águas é menor (cerca de 2 semanas) em comparação com Manguaba ( 2 meses), que praticamente não recebe aporte de água salgada. ${ }^{17}$

A lagoa Mundaú recebe o aporte da bacia hidrográfica do rio Mundaú, que drena uma área de aproximadamente $4.126 \mathrm{~km}^{2} \mathrm{e}$ abrange 30 municípios. Essa lagoa é mais afetada pela urbanização, principalmente da cidade de Maceió, com população de 900.000 habitantes. Já a lagoa Manguaba recebe o aporte dos rios Paraíba do Meio 
e Sumaúma, com bacias de drenagem de $3.330 \mathrm{~km}^{2}$ (20 municípios) e $406 \mathrm{~km}^{2}$ (3 municípios), respectivamente, sendo encontrados no seu entorno apenas cidades de menor porte, como Marechal Deodoro ( 45.000 habitantes) e Pilar ( 32.000 habitantes)..$^{21}$

\section{Amostragem e preparação das amostras}

Foram coletados, em março de 2007, 4 testemunhos de sedimento (28 a $47 \mathrm{~cm}$ de comprimento), sendo um na lagoa Manguaba (C03), 2 na lagoa Mundaú (C06 e C07) e um no leito do rio Mundaú (C08) (Figura 1S, material suplementar). Os testemunhos, obtidos através de amostrador por gravidade, foram seccionados a cada 2 ou $4 \mathrm{~cm}$ ainda no campo, sendo as subamostras armazenadas em recipientes apropriados e mantidas sob refrigeração. No laboratório, as amostras foram mantidas congeladas $\left(-20^{\circ} \mathrm{C}\right)$ e antes da extração foram secas em estufa $\left(60^{\circ} \mathrm{C}\right)$ até peso constante.

\section{Determinação de esterois}

Cerca de $5 \mathrm{~g}$ de sedimento seco foram submetidos à extração com $200 \mathrm{~mL}$ de uma mistura de diclorometano e metanol (9:1,v:v, marca J.T.Baker, grau pesticida), em aparelho Soxhlet, por $24 \mathrm{~h}$. Antes da extração, $10 \mu \mathrm{g}$ de androstanol ( $5 \alpha$-androstan-3 $\beta$-ol, marca Sigma, $98 \%$ de pureza) foram adicionados como padrão subrrogado. O volume do extrato bruto foi reduzido a $1 \mathrm{~mL}$ em TurboVap ${ }^{\circledR}$ e uma parte desse extrato concentrado foi saponificada a $85^{\circ} \mathrm{C}$ por $2 \mathrm{~h}$ usando-se uma solução de $\mathrm{KOH} 3 \mathrm{~mol} \mathrm{~L}^{-1}$ em solução $20 \%$ de água em metanol (J.T.Baker, grau pesticida). A fração neutra, contendo os esterois, foi recuperada com hexano e tratada com sulfato de sódio anidro, por 8 h, para remoção de traços de água. Os esterois foram separados em coluna de vidro contendo, da base para o topo, $11 \mathrm{~g}$ de sílica gel (70230 mesh, Merck), $1 \mathrm{~g}$ de alumina (70-230 mesh, Merck) - ambas 5\% desativadas com água ultrapura (Direct-Q, da Millipore ${ }^{\circledR}$ ) -, sulfato de sódio (ativado a $105^{\circ} \mathrm{C}$ ) e cobre metálico (ativado por lavagem com $\mathrm{HCl} 2 \mathrm{~mol} \mathrm{~L}^{-1}$ ). Os esterois foram isolados utilizando-se $50 \mathrm{~mL}$ de diclorometano/metanol 9:1 (v:v, J.T.Baker, grau pesticida), após passar pela coluna $75 \mathrm{~mL}$ de hexano (J.T.Baker, grau pesticida) e $55 \mathrm{~mL}$ de hexano/diclorometano 1:1 (v:v, J.T.Baker, grau pesticida) - essas duas frações, contendo, respectivamente, hidrocarbonetos alifáticos e aromáticos, foram guardadas para análise posterior. Os extratos purificados foram levados à secura sob fluxo de nitrogênio e submetidos à reação de derivação, com N,O-bis(trimetil-silil-trifluoroacetamida) (BSTFA; marca Supelco) a $85^{\circ} \mathrm{C}$ por $1 \mathrm{~h}$. O volume final do extrato foi aferido a $1 \mathrm{~mL}$ e 5,0 $\mu \mathrm{g}$ de colestano (Sigma, $98 \%$ de pureza) foram adicionados como padrão de quantificação.

A determinação quali-quantitativa dos esterois foi realizada por cromatografia em fase gasosa acoplada à espectrometria de massas (ThermoFinnigan modelo Focus-DSQ). Foi usada uma coluna capilar, tipo HP5MS, com $30 \mathrm{~m}$ de comprimento, $0,25 \mathrm{~mm}$ de d.i. e filme interno de $0,25 \mu \mathrm{m}$ de espessura. A injeção de $1 \mu \mathrm{L}$ foi feita sem divisão do fluxo (modo splitless), com válvula aberta após 1 min e com injetor a $280^{\circ} \mathrm{C}$. He ultrapuro foi usado como gás de arraste, a fluxo constante de $1,4 \mathrm{~mL} \mathrm{~min}^{-1}$. A programação de temperatura do forno foi a seguinte: início a $60{ }^{\circ} \mathrm{C}$, depois com rampa de $15{ }^{\circ} \mathrm{C}$ $\min ^{-1}$ até $250{ }^{\circ} \mathrm{C}$, passando a $1{ }^{\circ} \mathrm{C} \min ^{-1}$ até $300{ }^{\circ} \mathrm{C}$, com isoterma final nesta temperatura por $5 \mathrm{~min}$. $\mathrm{O}$ detector de massas operou no modo de ionização por impacto de elétrons a $70 \mathrm{eV}$, enquanto que a análise foi feita no modo de varredura (50-650 uma; taxa de 1,02 s), com as temperaturas da linha de transferência e da fonte iônica ajustadas para 300 e $200{ }^{\circ} \mathrm{C}$, respectivamente.

Os esterois considerados - coprostanol ( $5 \beta$-colestan-3 $\beta$-ol),

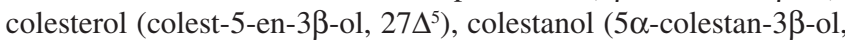
$\left.27 \Delta^{0}\right)$ e dinosterol $(4 \alpha-23,24$-trimetil-5 $\alpha$-colest-22(E)-en-3 $\beta$-ol,
$30 \Delta^{22}$ ) - foram identificados com base nos tempos de retenção e na comparação dos espectros de massas obtidos no próprio equipamento usando padrões autênticos (com exceção do dinosterol). A quantificação foi baseada na resposta relativa do íon $\mathrm{m} / z, 217$ do colestano (padrão de quantificação) para os íons $m / z 370$ (coprostanol), $m / z, 129$ (colesterol), $m / z, 215$ (colestanol) e $m / z, 358$ (dinosterol), e usando curva de calibração na faixa de 50 a $5000 \mathrm{ng} \mathrm{mL}^{-1}$ para os padrões disponíveis (marca Sigma, com pureza entre 95 e 99\%). Como não existe padrão comercial para dinosterol, a sua quantificação foi feita admitindo-se a mesma resposta relativa do $\mathrm{m} / \mathrm{z}, 358$ para o $\mathrm{m} / \mathrm{z}, 396$, do sitosterol (24-etilcolest-5-en-3ß-ol; marca Sigma, 98\% de pureza), também incluído na curva de calibração. Desta forma, os resultados de dinosterol são semiquantitativos e servem para comparar apenas as amostras analisadas.

O controle de qualidade do método analítico se deu através de: brancos de extração, que não mostraram contaminação das amostras durante o processamento das mesmas (Figura 2S, material suplementar); precisão do método, que foi melhor que $20 \%$ para 4 réplicas analisadas e, recuperação do padrão subrrogado androstanol, que foi de $99 \pm 35 \%$, considerada adequada para este tipo de análise. ${ }^{22} \mathrm{O}$ limite de quantificação foi calculado tendo como base a concentração do primeiro ponto da curva de calibração dos esterois e a massa média de sedimento extraída, ${ }^{23}$ sendo da ordem de $0,01 \mu \mathrm{g} \mathrm{g}{ }^{-1}$ para compostos individuais.

\section{RESULTADOS}

A Tabela 1 apresenta os resultados dos esterois para o total de 46 amostras analisadas nos 4 testemunhos coletados no CELMM, e na Figura 2S, material suplementar, encontra-se um cromatograma típico da distribuição dos esterois no sedimento. A concentração média de coprostanol na camada superficial $(0-2 \mathrm{~cm})$ dos testemunhos coletados foi de 2,02 $\pm 2,50 \mu \mathrm{g} \mathrm{g}^{-1}\left(0,15\right.$ a $\left.5,65 \mu \mathrm{g} \mathrm{g}^{-1}\right)$. A relativa variabilidade nos resultados na superfície do sedimento ocorreu devido às maiores concentrações nos testemunhos $\mathrm{C} 07$ (5,65 $\left.\mu \mathrm{g} \mathrm{g}^{-1}\right)$ e C06 (1,66 $\left.\mu \mathrm{g} \mathrm{g}^{-1}\right)$, ambos na lagoa Mundaú, em comparação com os níveis encontrados na lagoa Manguaba (testemunho C03: 0,63 $\mu \mathrm{g} \mathrm{g}^{-1}$ ) e no rio Mundaú (testemunho C08: $\left.0,15 \mu \mathrm{g} \mathrm{g}^{-1}\right)$. Com o aumento da profundidade nos testemunhos, observa-se um decréscimo das concentrações de coprostanol, sendo esse efeito mais acentuado no testemunho C07 ao longo dos primeiros $15 \mathrm{~cm}$ do sedimento (Figura 1). No geral, na base dos testemunhos ( $>30 \mathrm{~cm}$, exceto em C08), a concentração média de coprostanol de $0,09 \pm 0,04 \mu \mathrm{g} \mathrm{g}^{-1}\left(<0,01\right.$ a $\left.0,21 \mu \mathrm{g} \mathrm{g}^{-1}\right)$ pode ser considerada como o nível de referência (background) para o CELMM. É interessante notar que o testemunho C08 (rio Mundaú) apresentou, em geral, os menores valores para todos os esterois analisados. Possivelmente, isto é devido ao maior percentual de areia observado visualmente nessas amostras, mas esse resultado também reflete a menor tendência de acúmulo de matéria orgânica (tanto natural quanto antrópica) nesse rio.

Os esterois presentes em maiores concentrações nos sedimentos analisados foram o colesterol e o colestanol, com médias para as amostras de superfície de $9,15 \pm 7,34 \mu \mathrm{g} \mathrm{g}^{-1}\left(0,65\right.$ a $\left.17,28 \mu \mathrm{g} \mathrm{g}^{-1}\right)$ e 4,72 $\pm 3,47 \mu \mathrm{g} \mathrm{g}^{-1}\left(0,75\right.$ a $\left.8,67 \mu \mathrm{g} \mathrm{g}^{-1}\right)$, respectivamente, sendo os valores mais altos observados na lagoa Mundaú (Tabela 1). O colesterol é usualmente o esterol mais abundante em sedimentos marinhos pela sua ampla ocorrência no plâncton (fito e zoo) e animais, além das contribuições associadas a esgotos domésticos. ${ }^{6,24}$ Já o colestanol, embora também possa ter origem biogênica, ${ }^{25}$ é normalmente encontrado in situ como produto da redução microbiana do colesterol..$^{26,27}$ As variações nas concentrações de colesterol e colestanol ao longo do testemunho (Figura 1) são altamente correlacionadas entre si ( $\mathrm{r}=$ 
Tabela 1. Concentrações de esterois $\left(\mu \mathrm{g} \mathrm{g} \mathrm{g}^{-1}\right)$ e razões entre esterois selecionados em sedimentos do Complexo Estuarino Lagunar Mandaú-Manguaba

Testemunho Profundidade média $(\mathrm{cm})$ Coprostanol $\left(\mu \mathrm{g} \mathrm{g}^{-1}\right)$ Colesterol $\left(\mu \mathrm{g} \mathrm{g}^{-1}\right)$ Colestanol $\left(\mu \mathrm{g} \mathrm{g}^{-1}\right)$ Dinosterol $\left(\mu \mathrm{g} \mathrm{g}^{-1}\right)$ Colestanol/ Colesterol Cop/ (cop+colest $)^{\mathrm{a}}$ $\mathrm{C} 03$

7

11

15

19

21

25

27

29

33

35

39

41

C06

C07

C08

1
3

0,63

1,00

0,43

0,19

0,27

0,05

0,04

0,23

0,11

0,06

0,07

0,07

0,13

0,05

1,66

0,35

0,21

0,30

0,15

0,34

0,21

0,09

0,21

0,08

${ }^{\mathrm{b}}<$ LQ

5,65

1,63

1,12

0,84

0,32

0,35

0,23

0,33

0,23

0,20

0,16

0,03

0,10

0,15

0,19

0,35

0,37

0,05

0,05

0,13

0,07

$$
5,92
$$

6,11

3,50

3,24

2,25

1,31

1,43

1,84

1,51

2,27

1,96

1,75

1,62

1,87

17,28

3,96

1,42

2,01

1,63

1,85

0,90

1,58

1,09

0,26

$<$ LQ

12,74

3,28

2,48

1,67

1,81

1,80

1,51

1,42

1,05

0,91

0,86

0,08

0,81

0,65

0,41

0,75

0,72

0,52

0,35

0,58

0,47

3,19

4,02

2,05

1,57

1,08

0,71

0,89

0,92

0,70

0,97

0,86

0,73

1,10

1,45

6,27

3,39

1,86

2,63

1,62

1,66

1,03

1,37

1,03

0,25

0,09

8,67

3,83

3,24

2,37

1,91

1,52

1,39

1,51

0,84

0,65

0,65

0,17

0,62

0,75

0,54

0,88

0,77

0,47

0,33

0,41

0,37

\section{1,36}

2,35

1,53

1,53

1,02

0,58

0,37

0,67

0,58

0,90

0,61

0,69

0,58

0,63

3,01

1,22

0,54

0,75

0,85

0,78

0,45

0,64

0,56

0,28

0,05

2,90

1,23

1,45

1,40

1,14

0,58

0,74

0,86

0,46

0,35

0,37

0,15

0,08

0,21

$<$ LQ

0,10

0,19

0,21

0,09

0,17

0,19

0,54

0,66

0,59

0,49

0,48

0,54

0,62

0,50

0,46

0,43

0,44

0,42

0,68

0,78

0,36

0,86

1,31

1,31

1,00

2,81

0,90

1,15

0,87

0,95

0,95

0,68

1,17

1,31

1,42

1,06

0,84

0,92

1,06

0,80

0,71

0,76

2,25

0,77

1,16

1,31

1,16

1,06

0,91

0,92

0,71

0,79

0,17

0,21

0,17

0,11

0,20

0,07

0,05

0,20

0,14

0,05

0,08

0,09

0,10

0,04

0,21

0,09

0,10

0,10

0,09

0,17

0,17

0,06

0,17

0,24

n.c. ${ }^{\mathrm{c}}$

0,39

0,30

0,26

0,26 

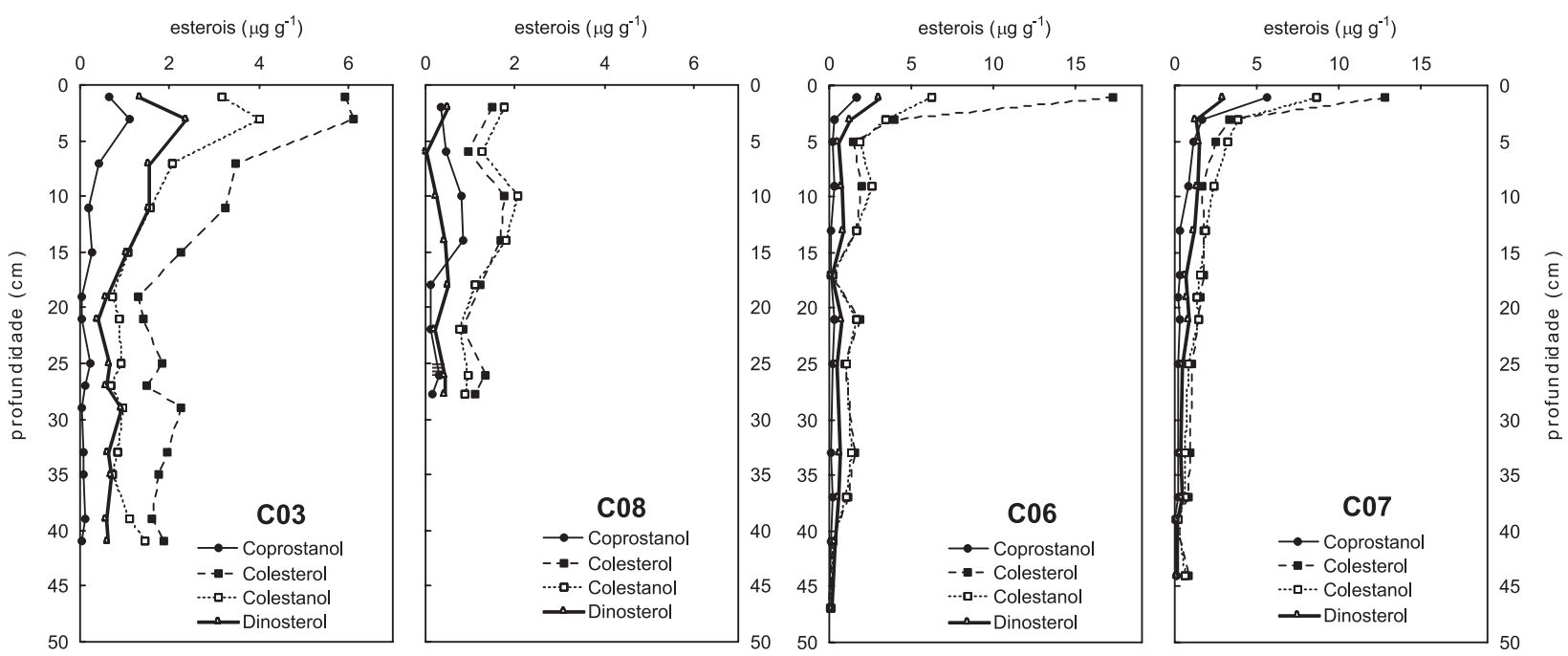

Figura 1. Perfis verticais das concentrações de coprostanol, colesterol, colestanol e dinosterol ao longo dos testemunhos coletados no CELMM. Os paineis C03 e C08 estão em escala de concentração inferior à dos paineis C06 e C07

$0,88 ; p<0,01)$, assim como as desses dois esterois para o coprostanol $(\mathrm{r}=0,73$ e 0,88 , respectivamente; $p<0,01)$.

O dinosterol é um biomarcador específico para organismos fitoplanctônicos, principalmente dinoflagelados, embora também seja produzido por diatomáceas. ${ }^{24} \mathrm{O}$ dinosterol apresentou concentração média de $0,77 \pm 0,61 \mu \mathrm{g} \mathrm{g}^{-1}\left(0,05\right.$ a 3,01 $\left.\mu \mathrm{g} \mathrm{g}^{-1}\right)$ nos sedimentos do CELMM. Os maiores valores foram encontrados na camada superficial do sedimento, com destaque para as lagoas Mundaú (C06: 3,01 $\mu \mathrm{g} \mathrm{g}^{-1}$; C07: 2,90 $\left.\mu \mathrm{g} \mathrm{g}^{-1}\right)$ e Manguaba (2,35 $\left.\mu \mathrm{g} \mathrm{g}^{-1}\right)$. As menores concentrações de dinosterol foram medidas no rio Mundaú e variaram na faixa entre 0,09 e $0,21 \mu \mathrm{g} \mathrm{g}^{-1}$ nas diferentes camadas sedimentares (Tabela 1). Com o aumento da profundidade, há decréscimo gradual nas concentrações do dinosterol em todos os testemunhos coletados, seguindo tendência observada para os outros esterois analisados (Figura 1).

\section{DISCUSSÃO}

\section{Nível de contaminação fecal no CELMM}

A entrada e a distribuição de esgotos domésticos em ambientes aquáticos podem ser reveladas usando coprostanol como traçador, mas há dificuldade em estabelecer um índice quantitativo de contaminação fecal com base nesse esterol. No caso de amostras de água, alguns trabalhos mostram que entre 30 e $400 \mathrm{ng} \mathrm{L}^{-1}$ de coprostanol correspondem a 1000 coliformes fecais por $100 \mathrm{~mL}$ de água (índice de balneabilidade na legislação brasileira), mas essa relação é altamente dependente da sobrevivência das bactérias sob diferentes condições ambientais, particularmente temperatura e salinidade.,.$^{4,28}$ A comparação entre colimetria e coprostanol no sedimento é ainda mais variável, e normalmente não existe correlação entre os dois parâmetros. ${ }^{10,29}$ De forma geral, concentrações a partir de $0,50 \mu \mathrm{g}$ $\mathrm{g}^{-1}$ de coprostanol sugerem sedimentos contaminados,,$^{30}$ embora valores da ordem de $0,10 \mu \mathrm{g} \mathrm{g}^{-1}$ sejam apontados como indicativos de contaminação fecal. ${ }^{31}$

Comparando as concentrações de coprostanol do presente trabalho com as obtidas em outras áreas no Brasil e no mundo (Tabela 2), pode-se considerar, a despeito das características sedimentológicas e hidrodinâmicas distintas em cada região, que os sedimentos superficiais da lagoa Mundaú (C06 e C07) apresentam contaminação moderada por esgotos domésticos. Já na lagoa Manguaba e no rio Mundaú, com níveis de coprostanol inferiores a $1 \mu \mathrm{g} \mathrm{g}^{-1}$, a contaminação por esgotos domésticos é ainda incipiente. A maior contaminação na lagoa Mundaú reflete o aporte de esgotos domésticos sem tratamento, oriundo da cidade de Maceió. Por outro lado, é interessante observar que embora o CELMM receba um grande aporte de esgotos ${ }^{21}$ e seja um ambiente que favoreça a retenção de materiais, devido à taxa de renovação da água relativamente baixa, ${ }^{17}$ as concentrações de coprostanol na lagoa Mundaú são inferiores a outras áreas listadas na Tabela 2 que são cronicamente impactadas por esgoto como, por exemplo, a baía de Guanabara e o estuário de Bilbao, na Espanha.

\section{Influência de processos diagenéticos sobre o acúmulo da contaminação fecal}

As concentrações relativamente baixas de coprostanol no CELMM poderiam resultar da degradação acelerada dos esterois pela alta atividade bacteriana típica de áreas tropicais, como já apontado para outro estuário no nordeste brasileiro. ${ }^{8}$ Caso isto ocorra, o registro sedimentar de coprostanol pode não ser representativo do histórico de acúmulo de esgotos no CELMM.

A razão colestanol/colesterol pode ser calculada para se avaliar o grau de transformação diagenética dos esterois em ambientes sedimentares. Isto porque na matéria orgânica "fresca", ou seja, aquela recentemente produzida por processos autotróficos, a razão colestanol/colesterol fica entre 0,1 e 0,5 devido à produção preferencial pela biota de esterois insaturados. ${ }^{26,32}$ Os altos valores para a razão colestanol/colesterol, com médias entre 0,5 e 1,0 para o conjunto de amostras de cada testemunho (Figura 3S, material suplementar), apontam a elevada influência diagenética nos marcadores utilizados nos sedimentos do CELMM. No entanto, é interessante notar que a variabilidade relativamente baixa (coeficiente de variação inferior a $25 \%$ ) dos dados ao longo dos testemunhos sugere que a degradação dos esterois é um processo pré-deposicional e, portanto, após o soterramento a relação entre os esterois permanece.

A razão coprostanol/(coprostanol+colestanol), originalmente proposta para indicar a contaminação fecal no caso de apresentar valores acima de $0,7,{ }^{16}$ também pode ser afetada por processos diagenéticos, devido à produção de colestanol in situ. Nos sedimentos do CELMM, o efeito da diagênese sobre esse índice ficou evidente, pois os valores foram sempre inferiores a 0,39 (Figura 3S, material suplementar), mesmo na lagoa Mundaú (C06 e C07), onde foram medidas as concentrações mais altas de coprostanol. Esse resultado corrobora o encontrado em outras áreas tropicais, ${ }^{4,9}$ mostrando a 
Tabela 2. Concentrações de coprostanol em sedimentos de diferentes regiões no mundo

\begin{tabular}{|c|c|c|c|c|}
\hline Localização & Camada sedimento $(\mathrm{cm})$ & Tipo de ambiente & Faixa de concentração $\left(\mu \mathrm{g} \mathrm{g}^{-1}\right)$ & Ref. \\
\hline \multicolumn{5}{|l|}{ BRASIL } \\
\hline Mundaú/Manguaba, AL & $0-2$ & Estuarino-lagunar & $0,15-5,65$ & Este estudo \\
\hline Rio Capibaribe, PE & $0-3$ & Estuarino & $0,52-7,31$ & 8 \\
\hline Florianópolis, SC & $0-2$ & Lagunar & $\mathrm{nd}^{\mathrm{b}}-2,88$ & 12 \\
\hline Lagoa dos Patos, RS & $0-2$ & Lagunar & nd-0,92 & 14 \\
\hline Baía de Ubatuba, SP & $0-3$ & Costeiro & nd- 0,27 & 13 \\
\hline Baia de Santos, SP & $\mathrm{ni}^{\mathrm{a}}$ & Costeiro & nd-8,51 & 33 \\
\hline Baía da Guanabara, RJ & $0-3$ & Costeiro & $0,33-40,00$ & 9 \\
\hline Baía de Sepetiba, RJ & $0-2$ & Baía semifechada & $0,01-0,42$ & 15 \\
\hline \multicolumn{5}{|l|}{ OUTRAS REGIÕES } \\
\hline Nova Iorque, EUA & $0-2$ & Costeiro & $0,56-5,20$ & 34 \\
\hline Bacia de Santa Mônica, EUA & $0-2$ & Costeiro & $0,50-5,10$ & 35 \\
\hline Baía de Narragansett, EUA & ni & Costeiro & $0,13-39,30$ & 36 \\
\hline Baía de Havana, Cuba & $0-3$ & Costeiro & $0,41-1,10$ & 16 \\
\hline Barcelona, Espanha & $0-3$ & Costeiro & $1,00-390$ & 16 \\
\hline Lagoa de Veneza, Itália & $\mathrm{ni}$ & Lagunar & $0,03-4,86$ & 37 \\
\hline Costa sudoeste, Taiwan & $0-4$ & Costeiro & nd- 0,82 & 38 \\
\hline Estuário Bilbao, Espanha & $0-0,5$ & Estuarino & $2,20-293$ & 30 \\
\hline Baía de São Vicente, Chile & $0-2$ & Costeiro & nd-7,30 & 39 \\
\hline Malásia e Vietnã & $0-5$ & Costeiro & $0,04-4,5$ & 4 \\
\hline Baía de Moreton, Austrália & $0-9$ & Baía semifechada & nd- 1,40 & 40 \\
\hline Estação Davis, Austrália & $\mathrm{ni}$ & Antártica & 1,28 & 41 \\
\hline Estação Ferraz, Brasil & $\mathrm{ni}$ & Antártica & $0,12-6,14$ & 42 \\
\hline Estação Rotera & ni & Antártica & 0,85 & 43 \\
\hline
\end{tabular}

${ }^{\mathrm{a}}$ ni $=$ não informado; ${ }^{\mathrm{b}}$ nd $=$ não detectado

limitação desse índice em regiões com temperaturas médias anuais elevadas. Destaca-se que o desvio-padrão relativamente baixo em cada testemunho do índice coprostanol/(coprostanol+colestanol) é consistente com a discussão anterior sobre o índice colestanol/ colesterol, que sugere limitada transformação diagenética da matéria orgânica após o seu soterramento.

\section{Registro histórico da contaminação fecal e de alterações ambientais}

Uma vez que não há indícios de degradação dos esterois após o soterramento no sedimento, pode-se considerar que o perfil de coprostanol no testemunho registra o histórico de contaminação fecal no CELMM. De fato, há uma relação significativa $(r=0,68$; $p=0,019)$ entre o acúmulo de coprostanol no sedimento e o crescimento populacional na cidade de Maceió. Isto possibilita se usar o coprostanol como uma abordagem alternativa para estimar a taxa de sedimentação no testemunho C07. Pode-se observar na Figura 2a que o aumento exponencial na concentração de coprostanol corresponde a uma população entre 700.000 e 800.000 habitantes. Por sua vez, essa população foi registrada a partir de 1996 (Figura 2b). Desta forma, considerando os primeiros $9 \mathrm{~cm}$ da camada sedimentar (profundidade média da camada onde a concentração de coprostanol atinge $0,84 \mu \mathrm{g} \mathrm{g}^{-1}$ ) e o período de 10 anos (1996-2006), a taxa de sedimentação no C07 equivale a 0,90 $\mathrm{cm}$ ano $^{-1}$. Este valor é muito próximo à taxa de sedimentação de $1,13 \mathrm{~cm}$ ano $^{-1}$ obtida para as camadas intermediárias do testemunho
C07 com base nos dados de Pb-210 (B. Knoppers, comunicação pessoal) e, portanto, nossa abordagem é válida.

Uma das principais consequências do lançamento de esgotos domésticos no CELMM é o aumento no nível de eutrofização do sistema, com reflexos sobre a composição, a abundância e a diversidade fitoplanctõnica. ${ }^{20}$ Neste sentido, é interessante observar a variação das concentrações de dinosterol e coprostanol no testemunho C07 (Figura 3). Na camada entre o fundo do testemunho até 12-14 cm há uma correlação linear entre dinosterol e coprostanol, sugerindo que o maior acúmulo do fitoesterol acompanhou o aumento na entrada de esgotos para a lagoa Mundaú. No entanto, a partir de $8-10 \mathrm{~cm}$ até a superfície do testemunho, o aumento nas concentrações de dinosterol foi proporcionalmente inferior ao do coprostanol. Uma explicação para esse comportamento é que houve uma mudança na estrutura da comunidade fitoplanctônica, sendo que as espécies que produzem dinosterol, principalmente dinoflagelados, passaram a ter menor importância a partir do período de maior acúmulo de esgotos domésticos, representado pelos primeiros $10 \mathrm{~cm}$ da coluna sedimentar (Figura 1). Essa hipótese é consistente com a predominância da diatomácea Skeletonema costatum na lagoa Mundaú nos últimos anos. ${ }^{19,20}$

\section{CONCLUSÕES}

No presente estudo foi avaliada a aplicação do coprostanol como indicador da evolução histórica da contaminação fecal de um sistema tropical caracterizado pela presença de múltiplas fontes de materiais 

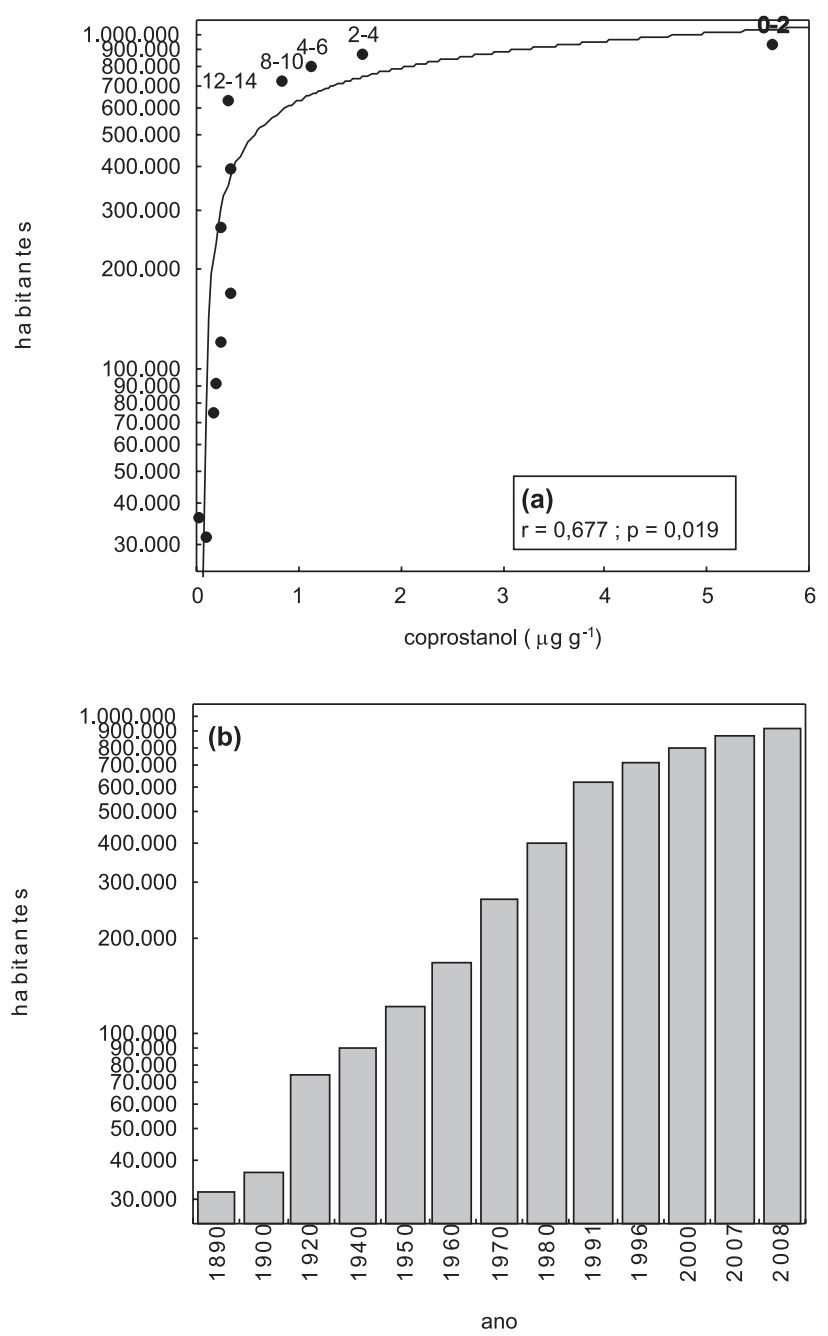

Figura 2. Perfil de coprostanol ao longo do testemunho C07 vs. o número de habitantes da cidade de Maceió nas últimas décadas (a) e variação da população da cidade de Maceió entre 1890 e 2008 (b)

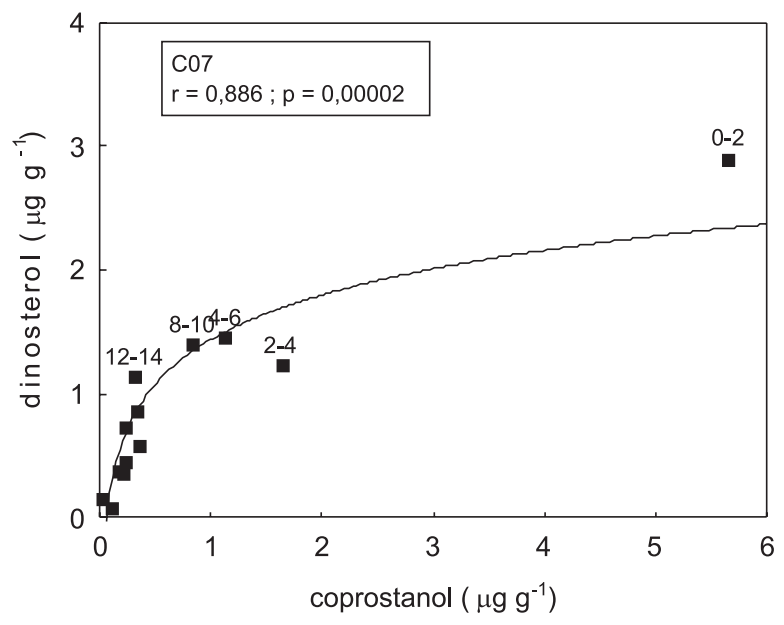

Figura 3. Correlação entre coprostanol e dinosterol no testemunho C07, na lagoa Mundaú

autóctonos e alóctonos, gradientes significativos nas suas propriedades físico-químicas e elevadas taxas de degradação bacteriana.

Como esperado, a lagoa Mundaú, devido à sua proximidade com a cidade de Maceió, apresentou os maiores níveis de contaminação fecal. Por outro lado, foi observado que a degradação bacteriana possivelmente causa uma diminuição dos valores absolutos de coprostanol frente ao esperado pelo aporte de esgotos para o sistema, e após o soterramento da matéria orgânica os esterois sugerem que não há transformações diagenéticas pós-deposicionais.

Desta forma, embora não seja possível usar o coprostanol para estabelecer um valor de referência para contaminação fecal que seja aplicável a diferentes sistemas tropicais, a distribuição espacial e temporal desse esterol, mais as informações obtidas através de razão entre compostos específicos, geram informações que permitem avaliar o processo histórico de acúmulo de esgotos domésticos para o sistema considerado.

Por fim, o perfil sedimentar de coprostanol e a evolução da população da cidade de Maceió permitiram estimar uma taxa de sedimentação de $0,90 \mathrm{~cm}^{2} \mathrm{no}^{-1}$ para a lagoa Mundaú.

\section{MATERIAL SUPLEMENTAR}

Está disponível gratuitamente em http://quimicanova.sbq.org.br, na forma de arquivo pdf. Apresenta: Figura 1S, com a localização dos testemunhos coletados no CELMM; Figura 2S, com exemplo de cromatogramas (fragmentogramas) parciais de um branco de análise e de uma amostra de sedimento e, Figura 3S, com as razões calculadas entre esterois específicos

\section{AGRADECIMENTOS}

Este estudo foi financiado pelo projeto POLCAMAR (processo n. 590002-2005-8), realizado dentro do convênio CNPq-MCT-BMBF de cooperação Brasil-Alemanha. Os autores agradecem ao Dr. P. R. P. Medeiros (LABMAR-UFAL) pelo apoio e disponibilização de infraestrutura para realização das coletas, e aos Drs. B. A. Knoppers, N. Brandini e E. Santos pela organização dos trabalhos de campo e processamento inicial das amostras, assim como a todos os outros pesquisadores e alunos, do Brasil e da Alemanha, que contribuíram para a realização do projeto.

\section{REFERÊNCIAS}

1. Laws, E. A.; Aquatic Pollution: an introductory text, $2^{\text {nd }}$ ed., John Wiley \& Sons: New York, 2000.

2. Group of Experts on Scientific Aspects of Marine Pollution. Em Protecting the oceans from land-based activities, GESAMP Report and Studies n. 71, 2001.

3. Leeming, R.; Nichols, P. D.; Water Res. 1996, 30, 2997.

4. Isobe, K. O.; Tarao, M.; Zakaria, M. P.; Chiem, N.; Minh, L. Y.; Takada, H.; Environ. Sci. Technol. 2002, 36, 4497.

5. Takada, H.; Satoh, F.; Bothner, M. H.; Tripp, B. W.; Johnson, C. G.; Farrington, J. W. Em Molecular markers in environmental geochemistry; Eganhouse, R. P. ed.; American Chemical Society: Washington, 1997, cap. 12.

6. Takada, H.; Eganhouse, R. P. Em Encyclopedia of Environmental Analysis and Remediation; Meyers, R. A., ed.; John Wiley \& Sons, Inc.: New York, 1998, cap. 8.

7. McCalley, D. V.; Cooke, M.; Nickless, G.; Water Res. 1981, 15, 1019; Nichols, P. D.; Leeming, R.; Rayner, M. S.; Latham, V.; J. Chromatogr., A 1996, 733, 497; Leeming, R.; Ball, A.; Ashbolt, N.; Nichols, P.; Water Res. 1996, 30, 2893.

8. Fernandes, M. B.; Sicre, M.-A.; Cardoso, J. N.; Macedo, S. J.; Sci. Total Environ. 1999, 231, 1.

9. Carreira, R. S.; Wagener, A. L. R.; Readman, J. W.; Estuar. Coast. Shelf Sci. 2004, 60, 587.

10. Costa, R. L.; Carreira, R. S.; Braz. J. Oceanog. 2005, 53, 157. 
11. Kawakami, S. K.; Dissertação de Mestrado, Universidade de São Paulo, Brasil, 1999; Martins, C. C.; Ferreira, J. A.; Taniguchi, S.; Mahiques, M. M.; Bícego, M. C.; Montone, R. C.; Mar. Pollut. Bull. 2008, 56, 1359; Santos, E. S.; Carreira, R. S.; Knoppers, B. A.; Braz. J. Oceanog. 2008, 56, 97; Cordeiro, L. G.; Carreira, R. S.; Wagener, A. L. R.; Org. Geochem. 2008, 39, 1097.

12. Mater, L.; Alexandre, M. R.; Hansel, F. A.; Madureira, L. A. S.; J. Braz. Chem. Soc. 2004, 15, 725.

13. Muniz, P.; Pires-Vanin, A. M. S.; Martins, C. C.; Montone, R. C.; Bicego, M. C.; Mar. Pollut. Bull. 2006, 52, 1098.

14. Martins, C. C.; Fillmann, G.; Montone, R. C.; J. Braz. Chem. Soc. 2007, $18,106$.

15. Carreira, R. S.; Ribeiro, P. V.; Silva, C. E. M.; Farias, C. O.; Quim. Nova 2009, 32, 1805.

16. Grimalt, J. O.; Fernandez, P.; Bayona, J. M.; Albaiges, J.; Environ. Sci. Technol. 1990, 24, 357.

17. Oliveira, A. M.; Kjerfve, B.; Estuar. Coast. Shelf Sci. 1993, 37, 575.

18. Souza, W W. F. L.; Machado, E. C.; Knoppers, B. A. Em Estuarine systems of the Latin American region (Regional Workshop V) and estuarine systems of the Arctic region: carbon, nitrogen and phosphorus fluxes - LOICZ Reports and Studies n. 23; Camacho-Ibar, V.; Dupra, V.; Wulff, F.; Smith, S. V.; Marshall Crossland, J. I.; Crossland, C.J ., eds.; LOICZ: Texel, 2002, cap. 5.

19. Magalhães, E. M. M.; Koening, M. L.; Sant'Anna, C. L.; Hoehnea 2004, 31,73 .

20. Melo-Magalhães, E. M.; Medeiros, P. R. P.; Lira, M. C. A.; Koening, M. L.; Moura, A. N.; Braz. J. Biology 2009, 69, 271.

21. Agência Nacional de Águas; Plano de Ação e manejo integrado do complexo estuarino-lagunar de Mundaú-Manguaba, Alagoas, Agência Nacional de Águas: Brasília, 2006.

22. Sauer, T. C.; Boehm, P. D.; Hydrocarbon chemistry analytical methods for oil spill assessments, MSRC Technical Report Series 95-032: Washington, 1995

23. Agência Ambiental Americana; Technical support document for the assessment of detection and quantitation approach, EPA-821-R-03-005: Washington, 2003.
24. Volkman, J. K.; Barret, S. M.; Blackburn, S. I.; Mansour, M. P.; Sikes, E. L.; Gelin, F.; Org. Geochem. 1998, 29, 1163.

25. Volkman, J. K.; Org. Geochem. 1986, 9, 83.

26. Wakeham, S. G.; Canuel, E. A. Em Handbook of Environmental Chemistry, Volume 2: Reactions and Processes $2(N)$; Volkman, J. K., ed.; Springer: Berlin, 2006, cap. 6

27. Sun, M.-Y.; Wakeham, S. G.; Org. Geochem. 1998, 28, 773.

28. Isobe, K. O.; Tarao, M.; Chiem, N. H.; Min, L. Y.; Takada, H.; Appl. Environ. Microbiol. 2004, 70, 814.

29. Nichols, P. D.; Leeming, R.; Rayner, M. S.; Latham, V.; Ashbolt, N.; Turner, A.; J. Chromatogr., A 1993, 643, 189.

30. González-Oreja, J. A.; Saiz-Salinas, J.; Mar. Pollut. Bull. 1998, 36, 868.

31. Choi, M.; Moon, H.-B.; Yu, J.; Kim, S.-S.; Pait, A. S.; Choi, H.-G.; Mar. Pollut. Bull. 2009, 58, 107.

32. Canuel, E. A.; Martens, C. S.; Org. Geochem. 1993, 20, 563.

33. Martins, C. C.; Gomes, F. B. A.; Ferreira, J. A.; Montone, R. C.; Quim. Nova 2008, 31, 1008

34. Hatcher, P. G.; McGilivary, P. A.; Environ. Sci. Technol. 1979, 13, 1225.

35. Venkatesan, M. I.; Kaplan, I. R.; Environ. Sci. Technol. 1990, 24, 208.

36. LeBlanc, L. A.; Latimer, J. S.; Ellis, J. T.; Quinn, J. G.; Estuar. Coast. Shelf Sci. 1992, 34, 439.

37. Fattore, E.; Benfenati, E.; Marelli, R.; Cools, E.; Fanelli, R.; Chemosphere 1996, 33, 2383.

38. Jeng, W.-L.; Wang, J.; Han, B.-C.; Environ. Pollut. 1996, 94, 47.

39. Mudge, S. M.; Seguel, C. G.; Mar. Pollut. Bull. 1999, 38, 1011.

40. Pratt, C.; Warnken, J.; Leeming, R.; Arthur, J. M.; Grice, D. I.; Environ. Sci. Technol. 2007, 41, 792.

41. Grenn, G.; Skerratt, J. H.; Leeming, R.; Nichols, P.; Mar. Pollut. Bull. 1992, 25, 293.

42. Martins, C. C.; Venkatesan, M. I.; Montone, R. C.; Antarct Sci, 2002, 14, 244.

43. Hughes, K. A.; Thompson, A.; Environ. Pollut. 2004, 127, 315. 


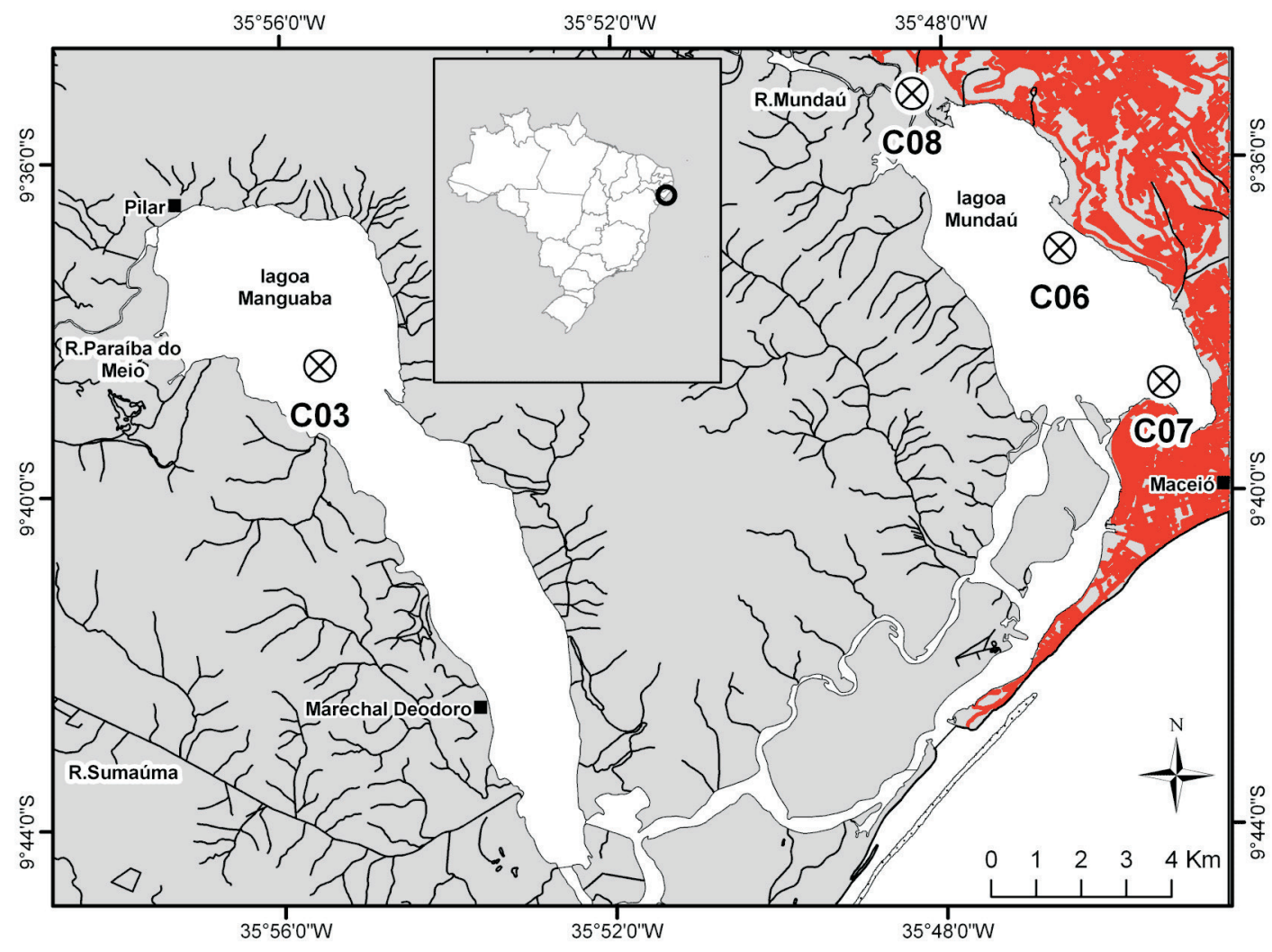

Figura 1S. Área de estudo e pontos de amostragens dos testemunhos de sedimento do CELMM. A cidade de Maceió está representada pela área em vermelho 


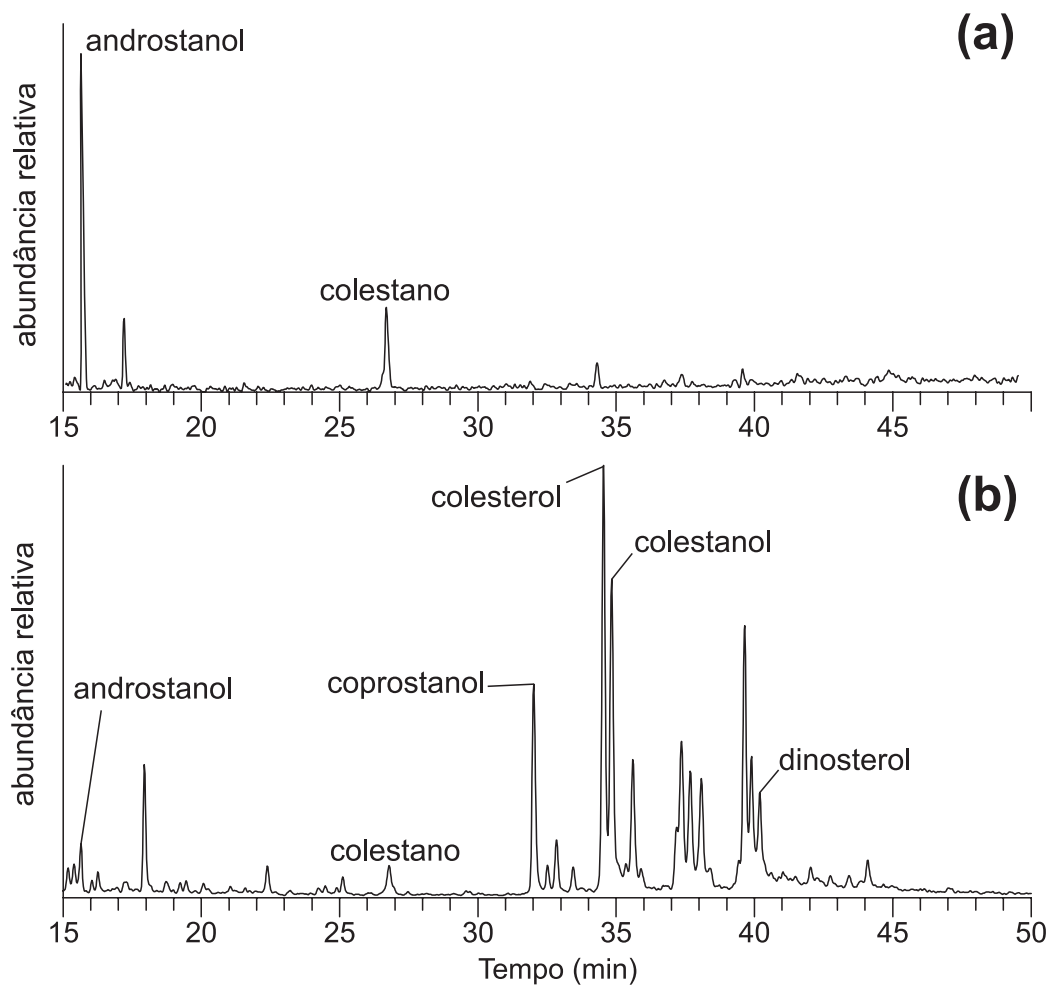

Figura 2S. Cromatogramas (fragmentogramas) parciais reconstituídos para o somatório dos íons dos esterois considerados: $\mathrm{m} / \mathrm{z} 129$ (colesterol), $\mathrm{m} / \mathrm{z} 215$ (colestanol), $\mathrm{m} / z 358$ (dinosterol) e m/z 370 (coprostanol). Em (a) um branco de laboratório e em (b) exemplo para a amostra 0-2 cm do testemunho C07. O padrão subrrogado androstanol e o padrão de quantificação colestano também são indicados

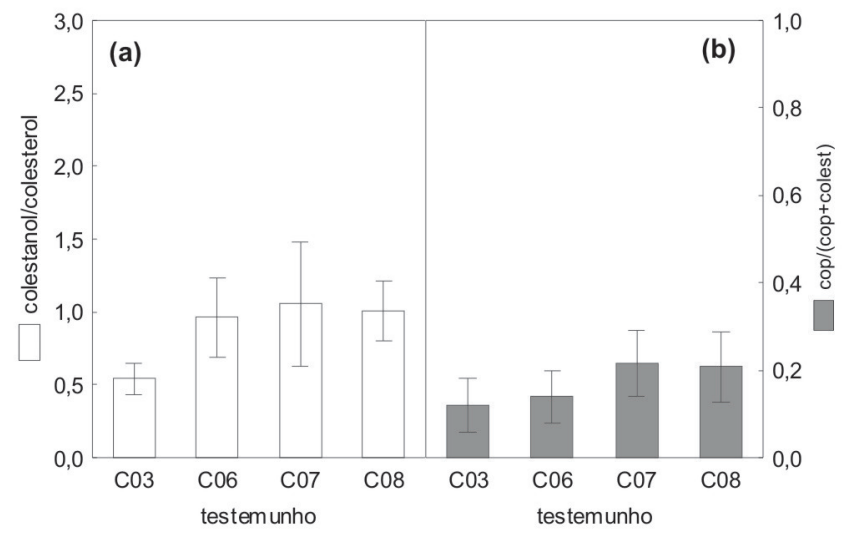

Figura 3S. Faixa de variação (média \pm desvio-padrão) para razões calculadas entre (a) colestanol/colesterol e (b) coprostanol/(coprostanol+colestanol) nos testemunhos de sedimento no CELMM 